\title{
Improved SURF Algorithm and Its Application in Seabed Relief Image Matching
}

\author{
Hong-Mei ZHANG ${ }^{1}$, Le YANG, Ming-Long $\mathrm{LI}^{1}$ \\ ${ }^{1}$ Department of Automation, School of Power and Mechanical Engineering \\ Wuhan University, Wuhan, China , e-mail: le_yang@whu.edu.cn
}

\begin{abstract}
The matching based on seabed relief image is widely used in underwater relief matching navigation and target recognition, etc. However, being influenced by various factors, some conventional matching algorithms are difficult to obtain an ideal result in the matching of seabed relief image. SURF(Speeded Up Robust Features) algorithm is based on feature points pair to achieve matching, and can get good results in the seabed relief image matching. However, in practical applications, the traditional SURF algorithm is easy to get false matching, especially when the area's features are similar or not obvious, the problem is more seriously. In order to improve the robustness of the algorithm, this paper proposes an improved matching algorithm, which combines the SURF, and RANSAC (Random Sample Consensus) algorithms. The new algorithm integrates the two algorithms advantages, firstly, the SURF algorithm is applied to detect and extract the feature points then to pre-match. Secondly, RANSAC algorithm is utilized to eliminate mismatching points, and then the accurate matching is accomplished with the correct matching points. The experimental results show that the improved algorithm overcomes the mismatching problem effectively and have better precision and faster speed than the traditional SURF algorithm.
\end{abstract}

\section{Introduction}

AUV (Autonomous Underwater Vehicle) is a type of unmanned underwater vehicle (UUV), Because of its high autonomy, passivity, invisibility, it has been widely used in the field of marine science and military[1]. With the development of the AUV, the image-matching algorithm based on seabed relief also becomes the research hot spot [2] Image matching algorithm is a key part of image matching navigation, and robust image matching algorithm is the basis of safe navigation. Under the influence of various factors, the conventional matching algorithm is prone to mismatching in seabed relief image matching, which brings security flaws to navigation, so it is of great significance to study the reliable image-matching algorithm.

Among the image matching algorithms, SURF algorithm as an improved algorithm of the SIFT (Scale-invariant feature transform ) algorithm, first proposed by Bay in 2006, due to its high efficiency and good robustness to rotation and scale, SURF is widely used in object recognition, 3D reconstruction and image matching[3-6]. Many scholars have studied the image matching algorithm based on SURF, Xie Yulai proposed underwater images real-time registration method based on SURF[3]; SU Kexin presented anti-viewpoint changing image matching algorithm based on SURF[4]; Yanwei Pang used fully affine invariant SURF for image matching[5]; ZHAO Lu-lu proposed an images matching algorithm based on SURF and fast approximate nearest neighbor search for that nearest neighbor matching of high-dimensional feature vector was low[6]. The SURF algorithm has achieved good results in a specific application. Accurate extraction of the image feature points is the key to ensure successful matching. However, in the practical application, the traditional SURF algorithm is prone to mismatching, especially in the area where the seabed features are not obvious.

RANSAC algorithm is a model parameter estimation method, first proposed by Fischler and Bolles in 1981[7], which can be used to deal with outliers in the data, and widely used in the purification of data. Qu Tianwei's research confirms that the RANSAC algorithm can reduce the interference of outliers and improve the matching effect [8]. In order to reduce the error matching, improve the performance of the SURF algorithm, and ensure the correct matching of the seabed relief image, comprehensively utilize the advantages of SURF algorithm and RANSAC algorithm to present an improved SURF algorithm. First, the matching image and reference image is preprocessed, then use the SURF algorithm to extract their feature points and pre-match, finally, the RANSAC algorithm is used to 
eliminate the mismatching points, and on this basis, the fast and accurate image matching is realized.

\section{Traditional SURF algorithm and RANSAC algorithm}

\subsection{SURF Algorithm}

Hessian matrix has good performance in experiment speed and precision. This matrix is widely used to determine the specific location and size of the scale, and SURF algorithm uses Hessian matrix as feature extractor. For any point $X=(x, y)$ in the image $I$ that needs to be matched, the Hessian matrix $H(X, \sigma)$ is defined by vector $\sigma$ as:

$$
H(X, \sigma)=\left[\begin{array}{ll}
L_{x x}(X, \sigma) & L_{x y}(X, \sigma) \\
L_{x y}(X, \sigma) & L_{y y}(X, \sigma)
\end{array}\right]
$$

Among them, $L_{x x}(X, \sigma)$ is the convolution of point $X$ in matching image $I$ and the value of second-order Gaussian Filter $\partial^{2} g(\sigma) / \partial^{2} x$; the meaning of $L_{x y}(X, \sigma)$ and $L_{y y}(X, \sigma)$ is similar to this.

Bay et al. [9] proposed a box type filter approximate to second order Gauss filter, and then use the integral image to expedite the convolution speed, and reduce the computational work. For a gray image, the value of any point $(x, y)$ in the integral image refers to the sum of the gray values of all the points in the rectangle region, which is formed from the upper left corner of the image to this point.

$$
I_{\Sigma}(x)=\sum_{i=0}^{i \leq x} \sum_{j=0}^{j \leq y} I(i, j)
$$

In the original image, expand the size of the box to get a different scale image pyramid, template filtering as shown in Figure 1.
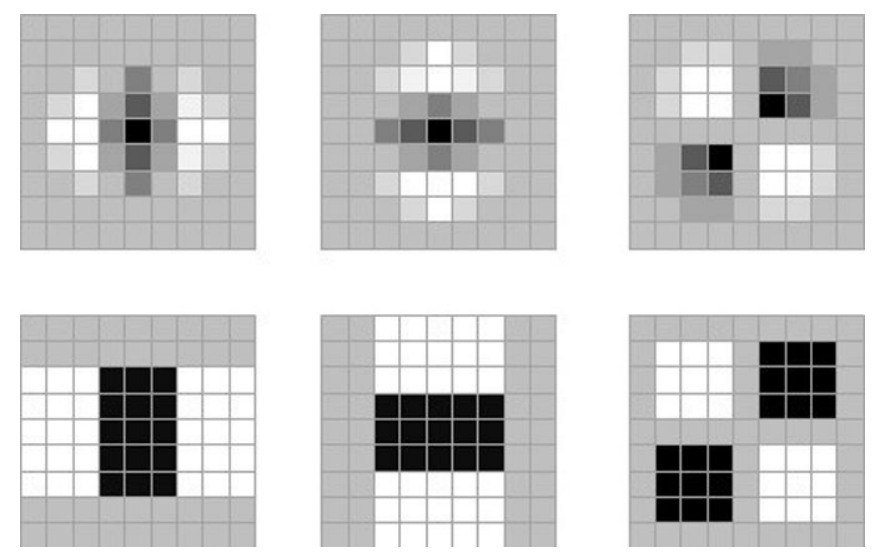

Fig.1 Box Filter

Box type filter in Figure 1 corresponds to second order Gauss filter, the value after Box filtering template respectively convoluting with the image is $D_{x x}$ 、 $D_{y y}$ and $D_{z z}$. The value of the Hessian matrix for each point in the scale space of the SURF operator, Bay et al. give an approximate formula as shown in formula (3).

$$
\text { delta }=D_{x x} D_{y y}-\left(0.9 D_{x y}\right)^{2}
$$

If the filter template size is $N \times N$, then the corresponding scale $s=\sigma=1.2 \times N / 9=1.2$. After using the Hessian matrix to find the extreme value, the nonmaxima suppression is carried out in the $3 \times 3 \times 3$ threedimensional domain. Only the feature points are larger or smaller than the 26 neighborhood values around the previous scale, then the next scale and current scale can be treated as candidate feature points, make a interpolation operation in scale space and image space to get stable feature points location and the scale value.

\subsection{RANSAC algorithm}

RANSAC algorithm is a resampling method, with minimal observation points to estimate the basic model parameters. For the basic assumption of the algorithm is: sample contains the correct data (inliers and can be described by the model of data), also contains abnormal data (outliers, deviated very far from the normal range, unable to adapt to the mathematical model of the data), that the data set contains noise. These abnormal data may be due to errors in measurement, incorrect assumptions, calculation and so on. In addition, RANSAC also assumes that, given a right set of data, there is a method to calculate the model parameters consistent with the data [10].

Its basic idea can be described as follows:

STEP1 : Randomly select the required minimum number of samples that can determine the model parameters.

STEP2: Calculate the model parameters. 
STEP3: Determine the number of points from all points that are in accordance with the prescribed tolerance.

STEP4: If the number of the correct points in the point set is more than the predetermined threshold value $\tau$, then use the determined interior points to re-estimate the model parameters.

STEP5: Otherwise, repeat 1 to 4 steps (up to $\mathrm{N}$ times).

Select enough number of iterations $N$ to ensure that at least one random sample set does not contain abnormal data and the probability $p \geq 0.99$. If it is assumed that the probability of any selected data point is the correct data for $\mathrm{u}$, then $v=1-u$ indicates the possibility of containing abnormal data. The minimum number of points required for the $N$ iterations is assumed to be $\mathrm{m}$, then

$$
1-p=\left(1-u^{m}\right)^{N}
$$

Transform to be

$$
N=\frac{\log (1-p)}{\log \left(1-(1-v)^{m}\right)}
$$

\section{The improved SURF algorithm}

\subsection{The Principle of Improved Algorithm}

The improved algorithm utilizes the advantages of the SURF algorithm and RANSAC algorithm, firstly, the feature points are extracted and pre-matched by SURF algorithm, then use RANSAC algorithm to eliminate the false matching points, lastly on this basis to carry on precise-matching. The principle of the algorithm is shown in Figure 2.

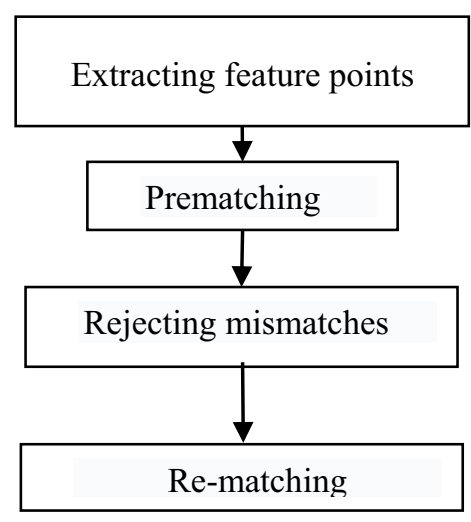

Fig.2 Improved algorithm principle diagram

\section{$4 \quad$ Experiment and analysis}

\subsection{Experimental image acquisition}

First, using side-scan sonar system to obtain seabed relief image, and the original image is preprocessed to obtain highquality seabed relief image for subsequent experiments.

Figure 3 (a) is the preprocessed image. In order to carry out the matching experiment, select the background image (a) in the black box as part of the target image to be matched, as shown in Figure 4 (b)
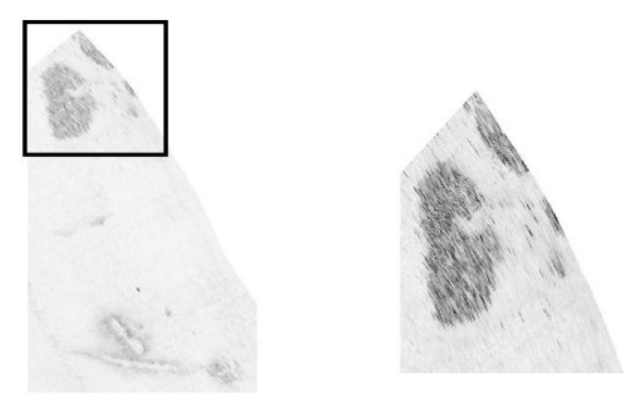
(a) background image
(b) target image

Fig.3 Experimental image selection

\subsection{SURF feature extraction}

Using Hessian matrix to extract the image's feature points, as shown in Figure 4.
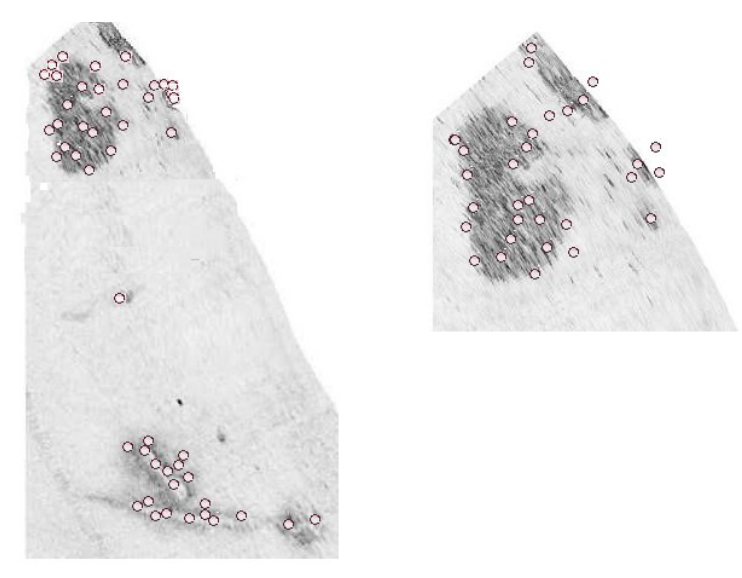

Fig.4 Image feature point extraction

It can be realized from Figure 6 that the Hessian matrix can effectively detect and extract the feature points in the area with high contrast and obvious features.

\subsection{Main direction determination}

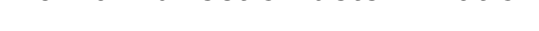


In order to distinguish the obtained feature points, the SURF algorithm uses the main direction of the feature points to describe the feature points.

In order to guarantee the rotation invariance, in SURF, there is no statistics of its gradient histogram, but the Haar wavelet features within feature point neighborhood are counted. That is feature points as the center, in the neighborhood of the calculated radius of $6 \mathrm{~S}$ (S is the scale value where the feature points lie.), the sum of the Haar wavelet responses of all points in the 60 -degree sector in the $\mathrm{X}$ and $\mathrm{Y}$ directions is statistically analyzed (Haar wavelet length is $4 \mathrm{~S}$ ). And the response value is assigned to the Gauss weight coefficient, which makes the response near the feature points is large, and the response far away from feature points is small, then use the response in the range of 60 degrees to form a new vector, traverse the entire circular area, the direction of the longest vector is selected as the main direction of the feature points. In this way, through the feature point calculation one by one, to get the main direction of each feature point, the process is shown in Figure 5.

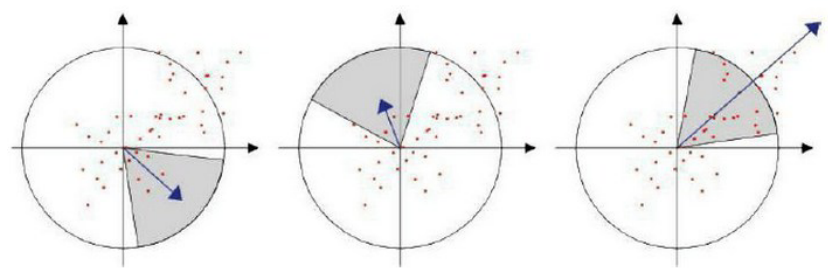

Fig.5 Determination of principal direction based on Haar wavelet

\subsection{Descriptor generation}

Feature point as the center, axis is fixed to the main direction of the main position, according to the main direction of the selected length for 20 s square area, and it was divided into $4 \times 4$ sub regions. In each sub region, some simple features are calculated. Now $d_{x}$ is defined as the Haar wavelet response in the horizontal direction, $d_{y}$ is the Haar wavelet response in the vertical direction. In order to enhance the robustness against the geometric deformation and the location error, a Gauss weight is added to the Haar wavelet response $d_{x}$ and $d_{y}$ at the feature points. Finally, the response of $d_{x}$ and $d_{y}$ in horizontal direction and vertical direction are accumulated separately in each sub region, then, $\left|d_{x}\right|$ and $\left|d_{y}\right|$ are accumulated separately to obtain the polarity of the intensity variation. The basic intensity image of each sub region is described by the following vector:

$$
V=\left(\sum d x, \sum d y, \sum|d x|, \sum|d y|\right)
$$

So,the length of descriptor vector formed by $4 \times 4 \mathrm{sub}$ region is 64 , and call this to be feature point's SURF descriptor.

\subsection{Feature matching}

The SURF descriptor of the feature point in the target image is pre-matched with the SURF descriptor of the corresponding block feature point in the background image [11]. Since the extracted feature points may be noise or nonfeature points, the initial match may not be correct. The matching result is presented in Figure6:

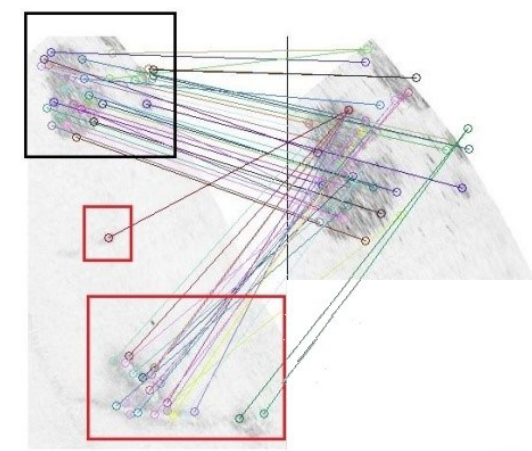

Fig.6 Feature points matching of SURF algorithm

In Figure 6, the black box part on the left side is the correct matching area, and the red box circle part is the mismatching area. Obviously, during the image matching process, the feature points in the red box will affect the success probability. For this reason, use RANSAC algorithm to remove the mismatching points, as shown in figure 7:

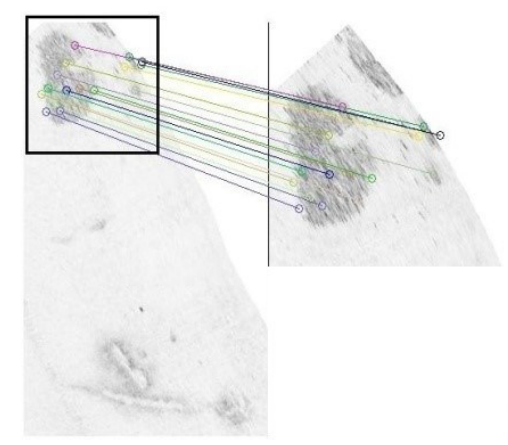

Fig.7: RACSAC algorithm removes the mismatching points

\subsection{Matching verification}

Randomly choose three pairs from the correct matching points to estimate the affine transformation matrix of feature 
point sequence. In this paper, RANSAC algorithm is used to estimate the affine transformation matrix $\mathrm{H}$ :

$$
H=\left[\begin{array}{lll}
h_{11} & h_{12} & h_{13} \\
h_{21} & h_{22} & h_{23} \\
h_{31} & h_{32} & h_{33}
\end{array}\right]
$$

Then the affine transformation matrix $\mathrm{H}$ is used to test whether the difference of each characteristic point position and the estimated position is within the tolerance range [12]. As follows:

$$
\begin{gathered}
\left(x_{i}^{\prime}-\frac{n_{11} x_{i}+n_{12} y_{i}+n_{13}}{h_{31} x_{i}+h_{32} y_{i}+h_{33}}\right)^{2}+\left(y_{i}^{\prime}-\frac{n_{21} x_{i}+n_{22} y_{i}+n_{23}}{h_{31} x_{i}+h_{32} y_{i}+h_{33}}\right)^{2}<\delta \\
i=1, \ldots, n
\end{gathered}
$$

When the number of matching points fit formula 8 is bigger than threshold $m$ and the difference between the feature point's position and the estimated position is less than the threshold $d_{\text {max }}$, and then believe the matching is successful. Otherwise, repeat the above procedure and re estimate the matrix $H$. If the number of corresponding feature points is less than four, or the matrix $\mathrm{H}$ is reestimated to be 100 times and there is no a constraint fits formula 8 , then the image matching is considered to be failure.

In Figure 6, the black box part on the left side is the correct matching area, and the red box circle part is the mismatching area. Compared with Figure 7, it can be found that there is no mismatching phenomenon after using the improved algorithm. The improved SURF algorithm uses SURF algorithm to extract feature points and pre match, and then use the RANSAC algorithm to eliminate the false matching point pairs, and on this basis to achieve precise matching. During the matching process, this method filtrates a large number of image's feature points, and rejects the mismatching points. The improved algorithm not only solves the mismatching problem in the traditional SURF algorithm, but also improves the problem of traditional SURF algorithm needs to match a large number of feature points. The simulation results show that the improved SURF algorithm has greatly improved the image matching accuracy. At the same time, compared with the traditional SURF algorithm, the matching error, matching efficiency and real-time performance are significantly improved. The performance comparison between the improved algorithm and the original algorithm is shown in Table 1.

\begin{tabular}{|c|c|c|}
\hline \multirow[t]{5}{*}{$\begin{array}{l}\text { Matching } \\
\text { method and } \\
\text { performance }\end{array}$} & $\begin{array}{c}\text { Number of } \\
\text { mismatching } \\
\text { feature } \\
\text { points }\end{array}$ & 19 \\
\hline & $\begin{array}{l}\text { Match } \\
\text { time/s }\end{array}$ & 25.21 \\
\hline & $\begin{array}{c}\text { Number of } \\
\text { feature } \\
\text { points }\end{array}$ & 15 \\
\hline & $\begin{array}{c}\text { Number of } \\
\text { mismatching } \\
\text { feature } \\
\text { points }\end{array}$ & 0 \\
\hline & $\begin{array}{l}\text { Match } \\
\text { time/s }\end{array}$ & 3.11 \\
\hline
\end{tabular}

Tab.1 Performance Comparison Before And After Improvement

\begin{tabular}{cc}
\hline Traditional SURF algorithm & Improved SURF algorithm \\
\hline Number of & 53 \\
feature & \\
points & \\
\hline
\end{tabular}

It can be seen from the Table 1 that whether in matching accuracy or matching time, the improved algorithm is better than the traditional SURF algorithm. The matching time is reduced to $12 \%$, and the matching efficiency is increased to $100 \%$.

\section{Conclusions}

SURF algorithm uses the concept of Haar feature and integral image, which can greatly speed up the matching speed; RANSAC algorithm can calculate the model parameters of the point set according to a set of sample points with abnormal data, and get the effective sample data. Due to the traditional SURF algorithm is prone to mismatching in the seabed relief image matching, the paper proposes an improved algorithm based on SURF algorithm and RANSAC algorithm, and applies it to the seabed relief image matching. The improved algorithm combines the advantages of SURF algorithm and RANSAC algorithm to improve the accuracy and robustness of the simulation, and the simulation results verify the effectiveness of the algorithm.

\section{Acknowledgment}

This work is partially supported by the National Natural Science Foundation of China (41376109) and National High Technology Research and Development Program of China (863 Program) (2009AA12Z311).

\section{References}

[1] Bo XU, Bai J L, Hao Y L, et al. The Research Status and Progress of Cooperative Navigation for Multiple AUVs[J]. Zidonghua Xuebao/acta Automatica Sinica, 2015, 41(3):445-461.

[2] Zhang H M, Zhao J H, Shao N. Underwater Match Navigation Based on Characteristic Seabed Relief Image[J]. Measurement \& Control Technology, 2011, 30(11):96-226.

[3] Xie Y, Li X, Lu J, et al. Underwater Images Real-Time 
Registration Method Based on SURF[J]. Journal of Computer-Aided Design \& Computer Graphics, 2010, 22(12):2215-2220.

[4] SU K, HAN G, SUN H. Anti-Viewpoint Changing Image Matching Algorithm Based on SURF[J]. Chinese Journal of Liquid Crystals and Displays, 2013, 4: 034.

[5] Pang Y, Li W, Yuan Y, et al. Fully affine invariant SURF for image matching [J]. Neurocomputing, 2012, 85: 6-10.

[6] Zhao L L, Geng G H, Kang L I, et al. Images matching algorithm based on SURF and fast approximate nearest neighbor search $[\mathrm{J}]$. Application Research of Computers, 2013, 30(3):921-923. (in Chinese)

[7] M.A. Fischler and R.C. Bolles. Random sample consensus: A paradigm for model fitting with applications to image analysis and automated cartography. Communications of the ACM, 24(6):381395, 1981.

[8] Wenchao L, Guodong L, Haiyan Y. Application of SIFT and advanced RANSAC algorithm on image registration[J]. Computer Engineering and Applications, 2013, 49(15): 147-149.

[9] Bay H, Tuytelaars T, Van Gool L. Surf: Speeded up robust features[M].Computer vision-ECCV 2006. Springer Berlin Heidelberg, 2006: 404-417.
[10] Zhang H, Shao N, Meng X, et al. 2010. Fast Image Matching Method and Its Applications in Underwater Positioning[J]. Electrical and Control Engineering (ICECE), International Conference on, 2010: 970 - 973.

[11] Tao W, Zhao J, Liu J, et al. Study on the side-scan sonar image matching navigation based on surf[C].Electrical and Control Engineering (ICECE), 2010 International Conference on. IEEE, 2010: 21812184.

[12] Chen F X, Wang R S. Fast RANSAC with Preview Model Parameters Evaluation[J]. Journal of Software, 2005, 16(8). 